\title{
DESAFIOS DE ACESSIBILIDADE E INCLUSÃO NO ENSINO SUPERIOR: UMA ANÁLISE SOB O OLHAR DO DESIGN UNIVERSAL PARA A MELHORIA NA EXPERIÊNCIA DE APRENDIZADO DOS DEFICIENTES VISUAIS
}

\author{
Íris Carlota dos Santos Arraes \\ Mestranda em Design - PUC-Rio \\ arraesics@gmail.com \\ Cláudia Mont'Alvão \\ Professora Associada \\ PPGDesign PUC-Rio \\ cmontalvao@puc-rio.br
}

Resumo: Apesar do grande numero de deficientes visuais no Brasil e os avanços no atendimento a esses usuários em diversos segmentos da sociedade, o acesso à universidade ainda é um problema. A presente pesquisa busca conhecer a trajetória acadêmica da pessoa com deficiência visual, desde o ensino fundamental até o superior, sob o olhar do Design Universal. Buscando um entendimento sobre esse cenário foi realizada uma análise documental das leis em vigor, que foram então relacionadas aos principais aspectos da Ergonomia física e cognitiva que estão inseridas no campo do Design Universal. Também foram considerados relatos sobre o tema postados em blogs de deficientes visuais, assim como notícias da mídia digital. Tais informações foram necessárias para que se esboçassem possíveis intervenções de Design que virem propostas projetuais que permitam um melhor acolhimento desses usuários.

Palavras-chave: Design universal; Deficientes visuais; Acessibilidade, Educação inclusiva; Ergonomia.

\begin{abstract}
Despite the large number of visually impaired people in Brazil and the development of special services for these users in different segments of society, access to university remains an issue. The present research intends to understand the academic path of people with visual impairment, from high school to university, under the perspective of Universal Design. To understand this scenario, a documental analysis of the applicable laws was carried out. They were then related to the main aspects of physical and cognitive ergonomics embedded in the Universal Design field. Visually Impaired Blog Reports were also considered, as well as digital media news. Such information was necessary to draft possible Design interventions that will enable these users to be better supported by the higher educational institutions
\end{abstract}

Key worlds: Universal design; Visually impaired; Acessibility; Inclusive Education; Ergonomics. 


\section{INTRODUÇÃO}

Há muito discute-se a inclusão social como pré-requisito dos direitos básicos e fundamentais do indivíduo como cidadão, e entre as inúmeras diversidades e deficiências, incluem-se nesse aspecto as pessoas com deficiência visual.

Estatísticas da OMS apontam que já existem 285 milhões de deficientes visuais no mundo, dentre os quais 39 milhões são totalmente cegos (WHO, 2014). Esta é uma população que cresceu exponencialmente nos últimos 10 anos, com o direito à educação inclusiva assegurado pela Declaração de Salamanca (UNESCO, 1994), que reafirma o direito à educação de todos os indivíduos.

Com este tratado, as nações - dentre elas o Brasil - ficaram sujeitas à elaboração de um Plano Nacional que assegurasse a educação básica aos seus cidadãos com necessidades especiais - o que veio a se efetivar em leis para a inclusão destas pessoas e, consequentemente, assegurar-lhes o direito ao aprendizado no ensino superior.

Mesmo com tantos tratados e leis, no Brasil, sabemos que a acessibilidade ao ensino não é realidade, podendo suas barreiras e dificuldades serem relatadas em Blogs e redes sociais pelos usuários que as encontram, e até mesmo em rápidas observações nos ambientes universitários.

A presente pesquisa tem por objetivo diagnosticar o panorama atual brasileiro no que se refere à acessibilidade para o candidato com deficiência visual ao ensino superior, assim como a sua posterior etapa - como aluno - à fim de elaborar diretrizes projetuais. Acredita-se que essas possam viabilizar uma melhora na interação destes usuários, com todo o tipo de assistência disponível que os levem a um melhor processo de aprendizado. Para tanto, vários aspectos teóricos do problema foram inicialmente pesquisados, tais como os conceitos e princípios do design universal, assim como aspectos da ergonomia física e cognitiva.

Embora as normas da Organização Internacional de Padronização (ISO 9999 2011) se refiram apenas à padronização da definição de produtos assistivos, descartando itens como assistência pessoal, cães-guia entre outras soluções não técnicas; a comunidade internacional tem reunido esforços em prol de colocar na agenda global a preocupação com estas pessoas com deficiência visual sob o escopo dos Direitos Humanos; e isso inclui toda e qualquer assistência prestada a elas. (BRASIL, 2006).

Verifica-se a relevância desta pesquisa para avanço na prática do designer como projetista integrante de projetos inclusivos no segmento da educação. 0 delineamento dos constrangimentos detectados a partir de estudos e projetos similares que incluem as pessoas com deficiência visual e suas consequentes análises, enriquecem o processo de elaboração de novos projetos de Design e Acessibilidade, indispensáveis para as instituições de ensino superior.

\section{DEFICIÊNCIA VISUAL: UMA CONTEXTUALIZAÇÃO}

De acordo com a última revisão da Classificação Internacional de Doenças em 2006, existem 4 diferentes níveis de acuidade visual (WHO, 2014). São elas: visão normal; deficiência visual moderada, deficiência visual severa e cegueira.

Agrupam- se sob o termo "baixa visão" os indivíduos do grupo de pessoas com deficiência visual moderada somado ao grupo de pessoas com deficiência visual severa, e aponta-se como principal causa para essas deficiências, erros de refração 
não tratados corretamente.

O grupo de pessoas com deficiência visual é aquele composto por cegos (total ausência de acuidade visual, que tem como maior de suas causas a catarata), e os de "baixa visão".

Segundo o Censo de 2010 (IBGE, 2012), são 45.606 .048 habitantes com algum tipo de deficiência no Brasil. Desses, $17,7 \%$ fazem a transição do ensino médio para o ensino superior, e somente $6,7 \%$ o concluem. Incluem-se nesse número, além dos habitantes com deficiência mental, motora e/ou auditiva, os que possuem alguma deficiência visual, que representam $20,1 \%$ da população.

Em 2010, 3,46\% eram já possuíam alguma deficiência visual severa, e 1,6\% eram totalmente cegos. A Deficiência visual teve maior prevalência entre todos os grupos de idade: $\mathrm{Na}$ faixa de 0 a 14 anos de idade, 5,3\% entre os investigados com alguma deficiência, eram pessoas com deficiência visual; na faixa de 15 a 64 anos, $20,1 \%$ e acima de 65 anos de idade, $49,8 \%$ - o que representa quase metade da população de deficientes investigada. Mesmo abraçando todas as deficiências, o Censo Universitário (INEP, 2014) aponta que em 2013 eram quase 30.000 alunos inscritos em cursos universitários, em sua maioria presenciais. Com esse levantamento, surgem as seguintes questões norteadoras desta pesquisa:

- Por qual motivo tão poucos deficientes visuais chegam ao ensino superior no Brasil?

- Existe possibilidade de igual aprendizado entre os alunos com deficiência visual e os alunos videntes nas instituições de ensino superior regular?

\subsection{A Importância da Acessibilidade no Desenvolvimento Escolar e Acadêmico}

A acessibilidade e a interação entre o ser humano e os sistemas são elementos fundamentais em práticas de projetos de Design e estudos em Ergonomia. Ao considerar a acessibilidade em sua forma mais abrangente nas instituições de ensino superior, desde o aspecto físico ao cognitivo para pessoas com deficiência visual; faz-se necessária a análise de como esses aspectos são trabalhados nas instituições de ensino básico, onde se inicia o processo de inclusão deste indivíduo na sociedade, tão imperativamente acordado na Declaração de Salamanca (UNESCO,1994).

Pensar na continuidade do desenvolvimento escolar desses cidadãos até o ensino superior, significa proporcionar-lhes o cumprimento de seus direitos fundamentais previstos no artigo 5어 da nossa Constituição Federal (BRASIL, 1988), e na Lei Brasileira de Inclusão da Pessoa com Deficiência (BRASIL, 2015).

Nesse contexto, observa-se a necessidade de estudos aprofundados em práticas projetuais de Design, que contribuam para o atendimento às pessoas com deficiência visual em sua trajetória escolar e universitária. Esses estudos devem considerar necessidades cognitivas e sociais, para que esses estudantes possam enriquecer seu aprendizado com conforto, autonomia e segurança também no ensino superior.

Compreender cada etapa desse processo é fundamental, visando responder as questões de pesquisa anteriormente colocadas, tais como:

- Os estudos sobre os diferentes níveis de deficiência visual; 
- Quais as barreiras e dificuldades relatadas pelo aluno com deficiência visual ao vivenciar a rotina no ensino superior;

- Como funcionam as instituições de ensino básico frequentadas por essas pessoas, sendo elas inclusivas ou não;

- Quais aspectos da ergonomia associados ao Design Universal precisam ser considerados e utilizados para que estes projetos e

- Quais possíveis intervenções de melhoria nas instituições de ensino superior, podem promover uma vivência e um processo de aprendizado eficaz por parte dos alunos com deficiência visual.

Para fins deste estudo, foram considerados os indivíduos integrantes do grupo de pessoas com deficiência visual severa e do grupo de pessoas sem nenhuma visão.

\subsection{Leis $\times$ Realidade: Como o Design Universal pode contribuir}

No âmbito do nosso país, o Plano Nacional de Educação inclui a Lei de Diretrizes e Bases (1996) que versa em seu parágrafo 1으, § 1ㅇ:

..." Esta Lei disciplina a educação escolar, que se desenvolve, predominantemente, por meio do ensino, em instituições próprias."

A Lei Brasileira de Inclusão da Pessoa com Deficiência (BRASIL, 2015), em seu capítulo IV relativo à educação incumbe ao poder público: avaliar:

Art.28. "Assegurar, criar, desenvolver, implementar, incentivar, acompanhar e

I- Sistema educacional inclusivo em todos os níveis e modalidades bem como o aprendizado ao longo de toda a vida;

V- Adoção de medidas individualizadas e coletivas em ambientes que maximizem o desenvolvimento acadêmico e social dos estudantes com deficiência, favorecendo o acesso, a permanência, a participação e a aprendizagem em instituições de ensino;

VI- Pesquisas voltadas para o desenvolvimento de novos métodos e técnicas pedagógicas, de materiais didáticos, de equipamentos e de recursos de tecnologia assistiva."

Entende-se que a adaptação à realidade da deficiência visual virou um grande desafio depois de implementada da Lei de Diretrizes e Bases da Educação Nacional (BRASIL, 1996), tanto para os órgãos governamentais relacionados como para as instituições de ensino. Muitas foram as modificações nas leis até a elaboração da Lei Brasileira de Inclusão à Pessoa com Deficiência (BRASIL, 2015), que em muito desenvolveu os aspectos referentes ao ensino superior para as pessoas com deficiência visual no Brasil. Porém, com a ausência do detalhamento e regulamentação acerca desta assistência no ensino básico, ainda em desenvolvimento, a legislação deixa a desejar quanto a sua eficácia.

O Projeto Livro Acessível (BRASIL, 2006) está disponível para a educação básica no Brasil, e abrange os ensinos infantil, fundamental e médio rede publica de ensino. Esse projeto assegura aos alunos com deficiência visual, materiais como laptops com o recurso MecDaisy de leitura em formato digital, livros em braile e ledores; ainda não existe nenhum projeto vigente que abranja esses recursos no 
ensino superior. Outro aspecto importante a ser considerado, é a distância entre o que diz a lei e o que de fato funciona no que tange a assistência às pessoas com deficiência visual nas instituições de ensino do Brasil. Fato, é que uma pessoa com deficiência visual é entendida como 'custo', seja para o governo ou para as instituições privadas.

Com o avanço da tecnologia em prol da pessoa com deficiência visual, é possível fazer o uso para analise de postagens em blogs e redes sociais das próprias, que estudam em instituições de ensino superior, como ferramentas para pesquisa acadêmica, (BUKVOVA; KALB; SCHOOP, 2010). Com a categorização do conteúdo postado e posterior classificação, é possível realizar um estudo qualitativo acerca da realidade do ensino superior em questões de acessibilidade para o aluno com deficiência visual. A consideração das dificuldades relatadas no conteúdo de postagens e notícias confirmam em parte as questões que norteiam essa pesquisa, citadas anteriormente.

Sendo "custo" para as instituições públicas ou privadas, a acessibilidade ainda não é completa e eficiente, como comprovam os relatos de alunos com deficiência visual que as frequentam e até mesmo notícias encontradas na imprensa e nas mídias digitais.

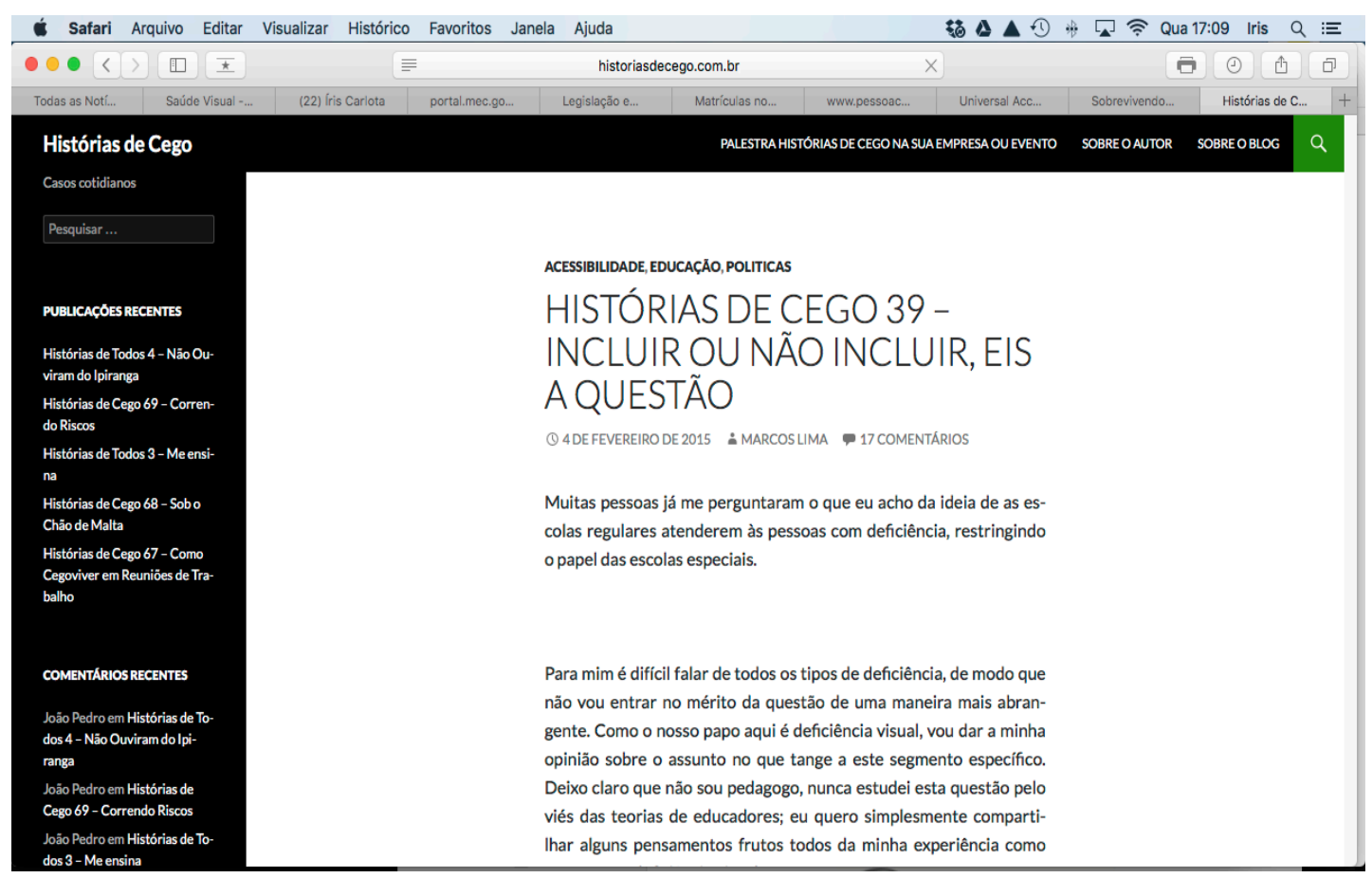

Figura 1: imagem de exemplo de Blog com relatos sobre a Educação Inclusiva 
globo.com g1 globoesporte gshow famosos \& etc vídeos

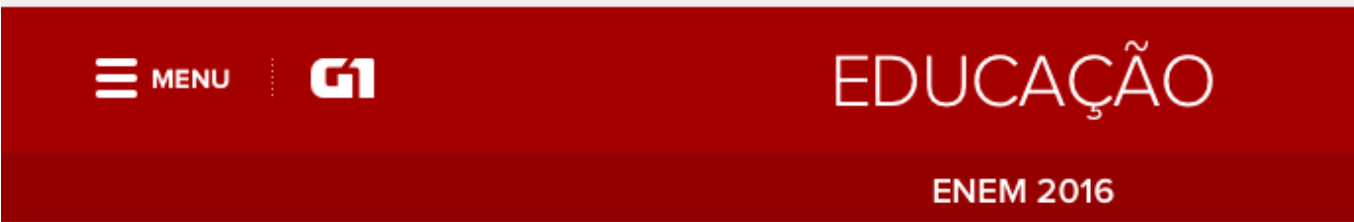

08/05/2016 07h23 - Atualizado em 08/05/2016 08h14

\section{MPF apura falta de acessibilidade em provas do Enem em 12 estados}

Para Procurador da República, MEC precisa prevenir este tipo de situação. Ministério diz que atendimentos específicos cresceram $226 \%$ em cinco anos.

Vanessa Fajardo

Do G1, em São Paulo

If FACEBOOK

$8^{+}$๑

O Ministério Público Federal (MPF) analisa 28 procedimentos que envolvem problemas de acessibilidade nas provas do Exame Nacional do Ensino Médio (Enem) em 12 estados brasileiros.

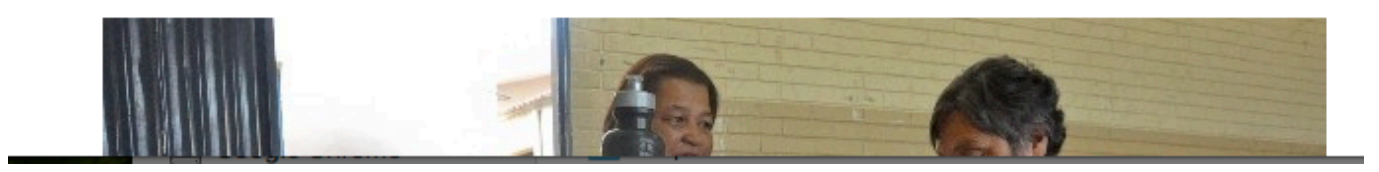

Figura 2: Notícia em mídia digital sobre a falta de acessibilidade em meio de ingresso para o Ensino Superior Brasileiro

\section{DESIGN, PROJETOS DE ACESSIBILIDADE E SUAS ABORDAGENS:}

Falar em Design relacionado a acessibilidade implica na consideração de diferentes abordagens. Por ser um campo de conhecimento muito amplo, os estudos relacionados ainda se encontram passíveis de críticas e discussões. Fato é que existe falta de consenso para uma padronização internacional do conceito de acessibilidade. Isto provavelmente se justifica pelas diferentes culturas e seus respectivos hábitos do cotidiano: o que representa acessibilidade na neve pode não representar no deserto, entre outra infinidade de exemplos interculturais que podem ser levantados.

Um estudo realizado no Instituto de Tecnologia Humana, Suécia (PERSSON, 2014), faz a análise de que as diferentes abordagens em Design revelam diferenças sutis no que tange a consideração do conceito de acessibilidade, mas que muito pode ser aprendido e somado a partir das premissas de cada metodologia e de suas perspectivas éticas e filosóficas.

Intimamente relacionada às diferentes abordagens do Design, encontra-se a Ergonomia que, segundo a Associação internacional de Ergonomia (IEA): "É uma disciplina científica relacionada ao entendimento das interações entre os seres humanos e outros elementos ou sistemas, e à aplicação de teorias, princípios, dados e métodos a projetos a fim de otimizar o bem-estar humano e o desempenho global 
do sistema" (ABERGO, 2007). Em outras palavras, a Ergonomia é a disciplina científica orientada a entender a relação dos seres humanos com uma tarefa a ser executada, seja essa tarefa relacionada a máquinas, trabalho ou ambiente; buscando através deste entendimento melhorias que as tornem compatíveis com as necessidades, e acessíveis dentro das diferentes limitações que possam vir a ser apresentadas, de maneira sistemática.

A Ergonomia abrange diferentes domínios de especialização, dentre eles estão:

\& Ergonomia Física: Relacionada a características da anatomia humana;

\& Ergonomia Cognitiva: Relacionada a processos mentais;

\& Ergonomia Organizacional: Relacionada à sistema sócio técnicos, incluindo estruturas organizacionais, políticas e de processos.

Para a pessoa com deficiência visual, é fundamental a consideração do conhecimento espacial e cognitivo, que são os principais viabilizadores da sua autonomia. Neste sentido que entra a contribuição dos domínios físicos e cognitivos, de especialização da Ergonomia.

Um exemplo desta consideração que vem a corroborar a importância de práticas em projetos é um estudo realizado na Universidade da Macedônia, na Grécia (KOUKOURIKOS; PAPADOUPOLOS, 2015), que confirmou que através de um dispositivo háptico - Novint Falcon - associado a um treinamento prévio, permitia que pessoas com cegueira, ou seja, com total ausência de acuidade visual, pudessem reconhecer rotas sem nenhum auxílio extra, explorando sua capacidade cognitiva no reconhecimento espacial obtendo sucesso.

A Ergonomia cognitiva, neste estudo, se relaciona com o processo mental de reconhecimento de espaço, tais como treinamentos e reconhecimentos de mapas mentais. Já a física, além dos aspectos relacionados à tarefa de manusear o dispositivo háptico, também se relaciona com o deslocamento urbano de fato. 0 reconhecimento mental de mapas é a prévia de como se dá o deslocamento físico no espaço urbano. A Ergonomia física se relaciona com muitos aspectos de acessibilidade nesse sentido. São viabilizadores às pessoas com deficiência visual na tarefa de deslocar-se pelas ruas: Os pisos táteis, os sinais sonoros, o cuidado na mudança de níveis entre calçadas e ruas, entre outros; estudados ergonomicamente.

Em outro estudo qualitativo, realizado em Botswana (PASHA ET HABULEZI, 2012), revela que para encontrar caminhos além do suporte do governo local em busca de melhorias no aprendizado de deficientes visuais, são necessários ajustes de quatro naturezas: físicos, curriculares, de práticas pedagógicas e de suporte familiar.

Sob a aplicação de técnicas como observações participantes, entrevistas em profundidade, e análise documental, foi possível neste estudo de caso analisar e compreender as necessidades dos alunos com deficiência visual de maneira aprofundada, sugerindo, categorizando e organizando mudanças que vão desde o aspecto físico ao cognitivo considerando, inclusive, fatores emocionais que influenciam indiretamente no aprendizado.

A Ergonomia organizacional neste estudo, entra como viabilizador estrutural de mudança encontrado nos resultados da pesquisa, organizando um processo 
participativo de melhorias educacionais aos deficientes visuais que inclui a participação da família, mudanças nos ambientes físicos das salas de aula inerentes à cada tipo de deficiência visual, acompanhamento extracurricular dos alunos, e treinamento específico aos docentes na universidade local, para que os alunos possam tenham condição de aprender melhor o conteúdo dado em sala de aula, melhorando assim os índices de aprovação em universidades, que era a principal questão levantada nesta pesquisa.

Aplicados ao ensino superior Brasileiro, os aspectos da Ergonomia física e cognitiva - por serem campos de conhecimento utilizados nas questões de interação do homem com tarefas e sistemas - adicionados às práticas de projetos em design, são capazes de direcionar os estudos relativos à acessibilidade que refinam as práticas de projeto. Estas práticas tornam possíveis a melhoria e o sucesso da experiência de aprendizado dos alunos com deficiências visuais.

Já existem iniciativas governamentais para perceber as diferenças e alinhar as diferentes abordagens do Design com as diferentes frentes que venham a surgir. Um exemplo de sucesso a ser citado é o Centro Nacional de Design Universal para Educação UDL Center, (2016) que, sob o olhar do Design Universal, oferece um conjunto de princípios para a elaboração de currículos que ofereçam oportunidades iguais de aprendizado a todos os indivíduos. Oferece um modelo de criação de metas para que todas as instituições interessadas possam alinhar acessibilidade com processos de aprendizado de forma única para cada indivíduo com suas diferentes particularidades, baseadas em três pilares. São eles:

\& Reconhecimento: O "Que" do processo de aprendizagem. Como categorizar o que é visto, sentido ou ouvido;

\& Redes estratégicas: O "Como" do processo de aprendizagem. O planejamento de como organizar e expressar as ideias;

\& Redes afetivas: O "Porque" do processo de aprendizagem. São as dimensões afetivas. Como motivar, engajar e tornar interessante no processo de aprendizagem.

Mesmo não explícitos, os domínios de especialização da ergonomia se encontram intimamente relacionados aos três pilares dos processos de aprendizado sugeridos pela UDL Center em termos cognitivos, assim como estarão presentes guiando a investigação de quais aspectos de acessibilidade não contemplam a deficiência visual relacionada ao ensino superior no Brasil, em termos físicos e cognitivos. Relacionados aos campos de conhecimento da Ergonomia, foram escolhidos os métodos do Design universal para pontuar as futuras estratégias nesta pesquisa.

No intuito de orientar uma ampla gama de disciplinas de design, incluindo ambientes, produtos e comunicações, um coeso estudo desenvolvido na Universidade do estado da Carolina do Norte (NCSU) - que resultou no Centro para o Design Universal (UDC, 1997) - regulou sete princípios básicos com a intenção de que os mesmos sejam aplicados para avaliar projetos, orientar o processo de design e educar os designers e consumidores sobre as características que permitam que produtos e ambientes possam vir a se tornar mais úteis e acessíveis. São eles: 
\& Uso Equitativo: O Design deve ser útil e comercializável para pessoas com diferentes habilidades;

\& Flexível no uso: O Design acomoda uma ampla gama de preferencias e capacidades individuais;

\& Uso simples e intuitivo: A utilização deve ser de fácil compreensão, independente da experiência prévia do usuário, conhecimentos, q linguísticas ou concentração atual nível;

\& Informação Perceptível: Comunica de forma eficaz ao as informações ao usuário independente de suas capacidades sensoriais ou condições ambientais;

\& Tolerância ao erro: O Design deve minimizar os riscos e consequências adversas de ações acidentais ou não intencionais;

\& Baixo esforço físico: Pode ser utilizado de forma eficiente, confortável e com um mínimo de fadiga;

\& Tamanho e espaço para o acesso e uso: Tamanho adequado e espaço são fornecidos para abordagem, alcance, manipulação e utilização, independentemente do tamanho do corpo, da postura ou mobilidade do usuário.

A preocupação do Design Universal em minimizar esforço físico, melhorar o acesso, fazer a utilização ser de fácil compreensão, e até mesmo categorizar e classificar os sete princípios, inclui também os processos da Ergonomia física, cognitiva e organizacional, correlacionando suas intenções em projetos de Design para melhorias em acessibilidade.

O Design universal e seus sete princípios, para os fins deste estudo, servirão de guia para as análises e considerações feitas a partir de técnicas próprias que serão aplicadas para extrair dados que venham a corroborar a proposta deste estudo qualitativo; no que tange os diferentes aspectos de acessibilidade encontrados na interação das pessoas com deficiência visual com o suporte que lhes é prestado, contribuindo assim com a melhoria na experiência de aprendizado no ensino superior.

\section{CONCLUSÃO}

Em breve análise documental das leis encontradas em vigor no Brasil, relacionadas à educação e à acessibilidade, fica nítida a falta de consenso e desorganização dos órgãos governamentais em diferentes aspectos, que vão da própria estrutura organizacional ao alinhamento da Lei de Diretrizes e Bases da Educação Nacional com a agenda internacional. Este fato por si, já provê consequências negativas nas instituições de ensino publico que dependem dos recursos do governo, em diferentes esferas - Federal e estadual - para investir em obras e tecnologias assistivas que viabilizem o aprendizado por parte dos alunos com deficiência visual. Já no ensino privado, onde as instituições possuem recursos próprios para investir e supostamente se encontram em melhores condições de investir em atributos físicos e tecnológicos, financeiramente, nem todos os candidatos com deficiência tem a oportunidade de ingressar.

O Design Universal em sua ampla abordagem com seus sete princípios, pode em muito contribuir na melhoria e otimização para a redução de custos em projetos 
relacionados à acessibilidade voltados à produtos assistivos e ao ambiente universitário. Para isto, faz-se necessária a investigação aprofundada através de técnicas como observações sistemáticas e assistemáticas acerca da rotina do estudante universitário com deficiência visual, assim como entrevistas semiestruturadas com os mesmos, com docentes e com núcleos de apoio ao aluno com necessidades especiais; a fim de compreender e analisar as barreiras que de fato interferem no seu aprendizado, de forma que o mesmo não alcance o desempenho em aprendizado de um estudante vidente.

Se o Design é Universal, precisa contemplar a experiência de aprendizado nas duas condições da mesma maneira, o que induz à conclusão de que não é possível a sua utilização para solucionar as questões governamentais, que influenciam diretamente na diferença da qualidade de aprendizado entre instituições públicas e privadas, por se tratar de uma estrutura organizacional. Neste aspecto, faz-se necessário considerar nas investigações futuras os aspectos da ergonomia física, relacionados à análise dos ambientes universitários; da ergonomia cognitiva, relacionados aos processos mentais do aprendizado destes estudantes, cabendo também à ergonomia organizacional o entendimento dos aspectos deficientes da estrutura governamental brasileira e seus conflitos nas leis de educação e acessibilidade, nas diferentes esferas.

\section{PRÓXIMAS ETAPAS}

Em pesquisa de campo na busca do objetivo desta pesquisa, para corroborar o estudo prévio apresentado neste artigo, serão investigadas através de entrevistas semiestruturadas as questões relacionadas à trajetória acadêmica estudantes com deficiência visual nos níveis propostos. Também serão investigados, através de observações assistemáticas e sistemáticas a rotina de deslocamento físico destes estudantes dentro da universidade, a fim de entender quais barreiras, no âmbito físico, atrapalham a experiência de aprendizado dos mesmos. Em seguida, com a extração destes dados, será feita a diagnose ergonômica deste estudo, sugerindo novas diretrizes viabilizadoras de melhorias na experiência de aprendizado ao ponto de alcançar o objetivo de ser equitativo entre os estudantes universitários deficientes visuais e os estudantes universitários videntes.

\section{REFERÊNCIAS}

ABERGO, Associação Brasileira de Ergonomia, 0 que é ergonomia? Disponível em: http://www.abergo.org.br/internas.php?pg=o_que_e_ergonomia Acesso em 29, Abr. 2016

BRASIL, Presidência da República, Casa Civil, Lei de Diretrizes e bases da educação Nacional 9394, 20 Dez. 1996. disponível em: http://www.planalto.gov.br/ccivil_03/leis/L9394.htm Acesso 11 Mar. 2016

BRASIL, Ministério da Educação, Secretaria de Educação Especial, Experiências Educacionais Inclusivas, Programa de Educação Inclusiva: Direito à diversidade, 2006 Disponível em:

http://portal.mec.gov.br/seesp/arquivos/pdf/experienciaseducacionaisinclusivas.pdf 
Acesso em: 14 Fev. 2016.

BRASIL, Presidência da República, Casa Civil, Lei Brasileira de Inclusão da Pessoa

Com Deficiência -13146 - 6, Jul. 2015 - disponível em:

http://www.planalto.gov.br/ccivil_03/_Ato2015-2018/2015/Lei/L13146.htm Acesso:

11 mar. 2016

BRASIL, Subsecretaria Nacional de Promoção dos Direitos da Pessoa com

Deficiência, Comitê de Ajudas Técnicas, Tecnologia Assistiva. Brasília: CORDE, 2009.

P. 138

BRASIL, Cartilha do Censo 2010 de Pessoas com Deficiência, Secretaria de Direitos Humanos da Presidência da república SDH/PR, Secretaria Nacional de Promoção dos Direitos da Pessoa com Deficiência SNDP, Coordenação Geral do Sistema de Informações Sobre a Pessoa com Deficiência, 2012.

BRASIL. Constituição (1988). Atos decorrentes do disposto no § 3ㅇ do art. 5이 da Constituição, de 9 de novembro de 1995.

BUKVOVA, Helena; KALB, Hendrik; SCHOOP, Erik, What we blog? A Qualitative Analisys of Researchers' Weblogs, Chair of Business Informations, esp. Informations Management, Faculty of business and Economics, Dresden Saxony, German

Conferência mundial sobre necessidades educativas especiais; SALAMANCA, Espanha 7-10 Jun. 1994. Declaração de Salamanca e Enquadramento da Ação nas Áreas de Necessidades Educativas Especiais, UNESCO 1994. Disponível em: http://portal.mec.gov.br/seesp/arquivos/pdf/salamanca.pdf

IBGE, CENSO Demográfico 2010, Características gerais da população, religião e pessoas com deficiência, Instituto Brasileiro de Geografia e Estatística 2012 disponível em:

http://biblioteca.ibge.gov.br/visualizacao/periodicos/94/cd_2010_religiao_deficienc ia.pdf Acesso 1 Jun. 2016

INEP, Instituto Nacional de Estudos e Pesquisas Educacionais Anísio Teixeira, Censo da Educação Superior, 09, Set. 2014, disponível em:

http://portal.inep.gov.br/visualizar/-/asset_publisher/6AhJ/content/matriculas-noensino-superior-crescem-3-8 Visualizado em 01, Jun. 2016

International Organization for Standardization , ISO 9999/2011, Assistive Products for Persons with Disability: Classification and Terminology Disponível em: http://www.iso.org/iso/catalogue_detail.htm?csnumber=50982 Acesso: 1 Jun. 2016 KOUKORIKOS, P; PAPADOUPOULOS, K - Development of Cognitive Maps by Individuals with Blindness Using a Multisensory Application - Department of Educational and social policy, University of Macedonia. $6^{\text {th }}$ International Conference on Software Development and Technologies for Enhancing Accessibility and Fighting Infoexclusion (DSAI 2015)

NACIONAL CENTER ON UNIVERSAL DESIGN FOR LEARNING, About UDL, Disponível em : http://www.udlcenter.org/aboutudl Acesso em 01 Jun. 2016

PERSSON, H. 2014 - Universal design, inclusive design, accessible design, design for all: different concepts - one goal? On the concept of accessibility: historical, 
methodological and philosophical aspects, Universal Access in the Information Society, November 2015, Volume 14, Issue 4, pp 505-526.

PASHA, TN; HABULEZI, J - Provision of learning support to learners with visual impairment, Department of inclusive Education, University of South Africa. International Conference on Education and Educational Psychology (ICEEPSY 2012) THE CENTER FOR UNIVERSAL DESIGN, The Principles of Universal Design, Version 2.0. Raleigh, NC: North Carolina State University, 1997. Disponível em: http://www.ncsu.edu/ncsu/design/cud/pubs_p/pud.htm - Acesso em 07.Mai.2016 WORLD HEALTH ORGANIZATION, Statistics for Visually Impairment, Fact Sheet № 282, 2014. Disponível em: http://www.who.int/mediacentre/factsheets/fs282/en/\#. Acesso em 29 Abr 2016. 